\section{Strangulated appendiceal intussusception caused by isolated endometriosis}

\author{
Seokyoun Lee', Junhee Lee ${ }^{2}$, Keunyoung Kim ${ }^{3}$ \\ 'Department of Surgery, Wonkwang University Sanbon Hospital, Wonkwang University School of Medicine, \\ Gunpo, Korea \\ ${ }^{2}$ Department of Emergency Medicine, Wonkwang University Sanbon Hospital, Wonkwang University School \\ of Medicine, Gunpo, Korea \\ ${ }^{3}$ Department of Surgery, Wonkwang University Hospital, Wonkwang University School of Medicine, Iksan, \\ Korea
}

A 33-year-old female was admitted to our emergency department for right lower quadrant abdominal pain, which was not associated with her menstrual cycle, and nausea, which had persisted for 3 days. She denied any prior history of abdominal pain, dysmenorrhea, menorrhagia, or dyspareunia. The patient had no family history of endometriosis. Abdominal computed tomography revealed appendiceal intussusception (AI) and invagination of the periappendiceal fat into the cecum (Fig. 1). She underwent laparoscopic partial cecectomy with the presumed diagnosis of Al (Fig. 2). The ovaries and fallopian tubes were normal, with no endometrial spot. The inverted appendix was completely necrotic (Fig. 3). Final surgical pathology was positive for gangrenous intussusception of the appendix, with endometrial deposits (Fig. 4).

Adult colonic intussusception is caused by either malignant tumors (64\%-87\% cases) or benign tumors (29\%-33\%). The most common causes are adenocarcinomas and lymphomas among malignant tumors and lipomas, leiomyomas, adenomatous polyps, and endometriosis among benign tumors. ${ }^{1} \mathrm{Al}$ is extremely rare, with reported rates of $0.01 \%$ in patients who undergo appendectomy. ${ }^{2} \mathrm{Al}$ secondary to endometriosis is also extremely rare, with less than 30 cases reported. However, those cases involved endometriosis on other organs and the appendix. Only three cases involving isolated appendiceal endometriosis are reported in the literatures. ${ }^{3}$ Patients with $\mathrm{Al}$ can range from being asymptomatic to presenting symptoms mimicking acute appendicitis. ${ }^{4}$ Preoperative diagnosis is difficult owing to its rarity. Although endometriosis is reported to be the lead point of $\mathrm{Al}$, there are limited descriptions on imaging findings. Computed tomography findings include an enlarged appendix with an inverted appendiceal tip or a tumor protruding into the

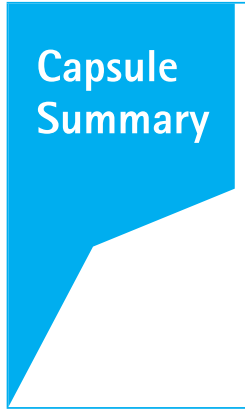

What is already known

Appendiceal endometriosis can present strangulated intussusception.

What is new in the current study

Appendiceal intussusception secondary to endometriosis should be considered in the differential diagnosis when female patients of reproductive age present with clinical symptoms of acute appendicitis.
elSSN: 2383-4625

Received: 16 March 2020

Revised: 18 May 2020

Accepted: 16 June 2020

Correspondence to: Seokyoun Lee Department of Surgery, Wonkwang University Sanbon Hospital, Wonkwang University School of Medicine, 321 Sanbon-ro, Gunpo 15865, Korea E-mail: necuri@wku.ac.kr ORCID https://orcid.org/0000-0001-7245-8449

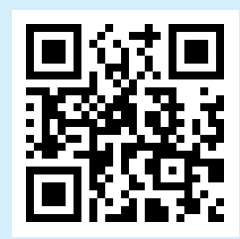

Lee S, Lee J, Kim K. Strangulated appendiceal intussusception caused by isolated endometriosis. Clin Exp Emerg Med 2021;8(3):246-248. https://doi. org/10.15441/ceem.20.026

This is an Open Access article distributed under the terms of the Creative Commons Attribution Non-Commercial License (https:// creativecommons.org/licenses/by-nc/4.0/). 

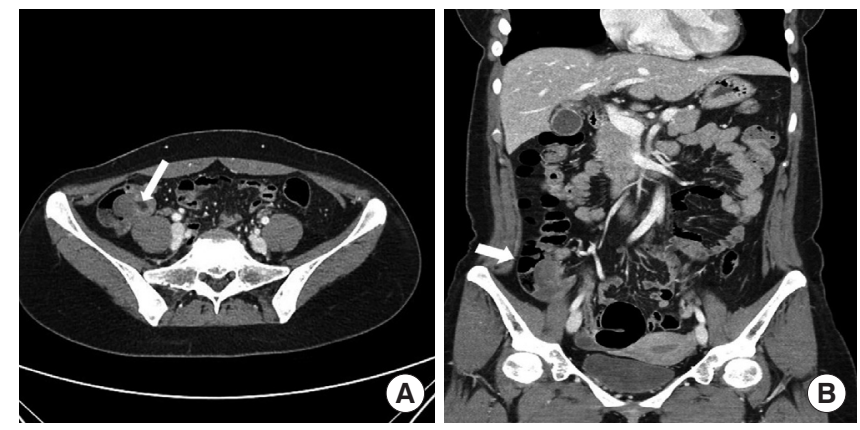

Fig. 1. The abdominal computed tomography. (A) Axial view and (B) coronal view. These images show a low-density tubular structure $(5 \times 1.6$ $\mathrm{cm}$ in diameter) in the cecum near the appendiceal orifice, and focal invagination of the peri-appendiceal fat to the cecum (white arrow).

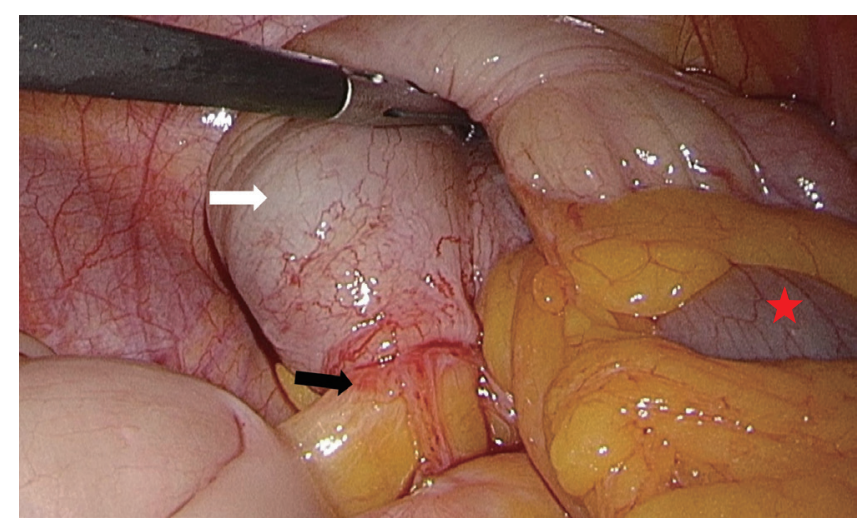

Fig. 2. Surgical findings. During laparoscopic exploration, the appendix was not macroscopically visible in the right iliac fossa, but it was located in the cecum (white arrow), which showed no possibility for reduction (black arrow). Terminal ileum denoted by asterisk.

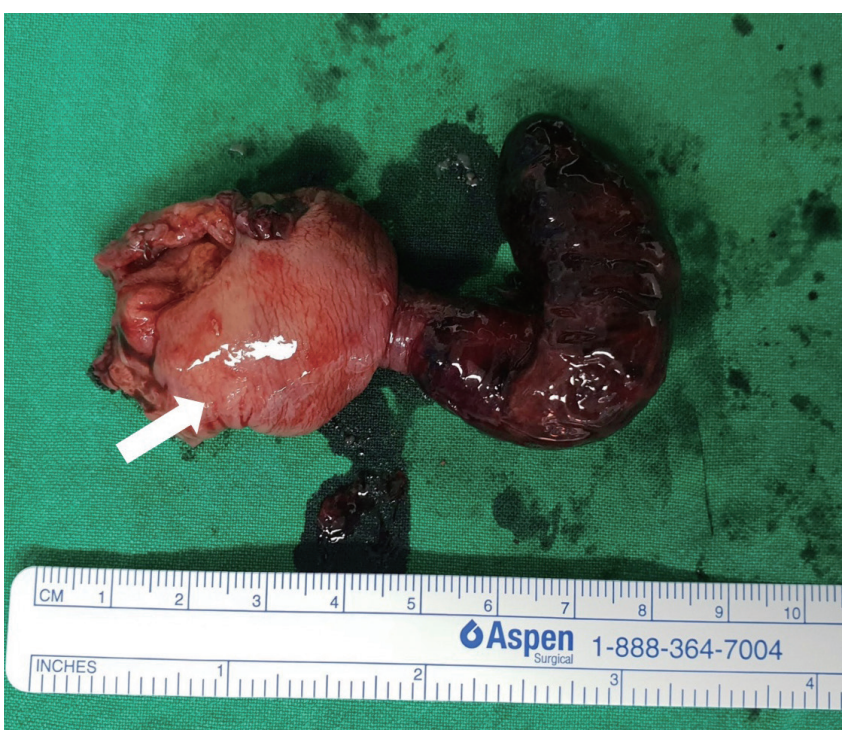

Fig. 3. Surgical specimen. Appearance of the specimen resected by laparoscopic partial cecectomy. The inverted appendix ( $70 \times 15 \mathrm{~mm}$ in size) is completely necrotic at the appendiceal orifice (inside the aspect of the cecum denoted by a white arrow).

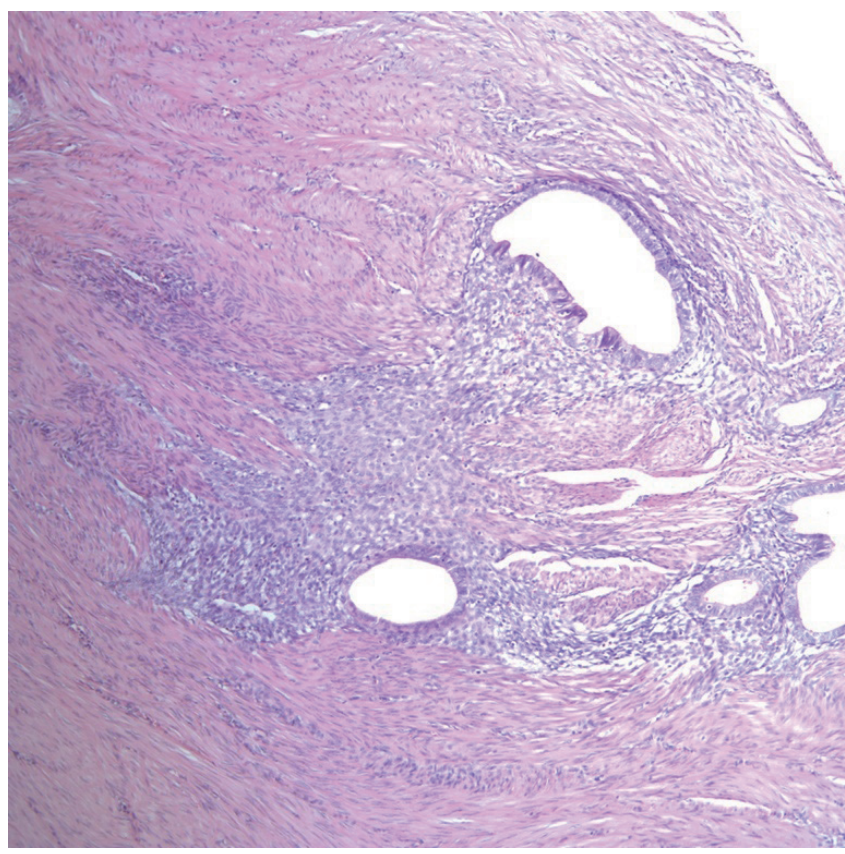

Fig. 4. High-power view of endometriosis within the appendix. Endometrial glands and stroma within the muscular layer, around the appendicular mucosa (hematoxylin and eosin, $\times 200$ ).

cecum. ${ }^{5}$ This case highlights that isolated endometriosis without involvement of reproductive organs can present as a strangulated Al. Al secondary to endometriosis should be considered in the differential diagnosis when female patients of childbearing age present with clinical symptoms of acute appendicitis and radiologic findings of intussusception, even before obtaining a pathological result.

\section{CONFLICT OF INTEREST}

No potential conflict of interest relevant to this article was reported.

\section{ACKNOWLEDGMENTS}

This paper was supported by Wonkwang University in 2020.

\section{REFERENCES}

1. Lee SY, Park WC, Lee JK, Kang DB, Kim Y, Yun KJ. Laparoscopic treatment of adult sigmoidorectal intussusception caused by a mucinous adenocarcinoma of the sigmoid colon: a case report. J Korean Soc Coloproctol 2011;27:44-9.

2. Collins DC. 71,000 Human appendix specimens. a final report, summarizing forty years' study. Am J Proctol 1963;14:265-81. 
3. Manoharan B, Haider AS, Samira AM, Bharathan B, ParraBlanco A. Isolated appendiceal endometriosis resulting in intussusception. Oxf Med Case Reports 2018;10:349-52.

4. Salehzadeh A, Scala A, Simson JN. Appendiceal intussusception mistaken for a polyp at colonoscopy: case report and review of literature. Ann R Coll Surg Engl 2010;92:W46-8.
5. Hanly AM, Ryan EM, McNamara DA. Appendiceal intussusception and endometriosis mimicking colorectal cancer. Colorectal Dis 2011;13:225-6. 